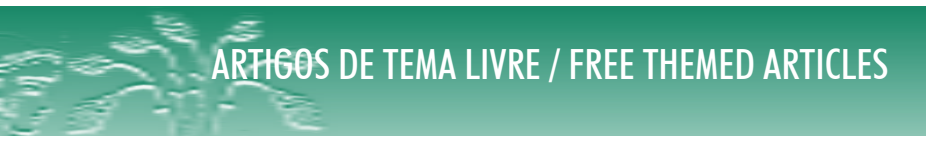

DOI: 10.12957/demetra.2018.28663

\title{
O Marco de Referência de Educação Alimentar e Nutricional para Políticas Públicas no Brasil no contexto do atendimento nutricional
}

\section{The FoodandNtritionEdrationFranenak for PublicPdicesin the context of nutritional care in Brazil}

Irene Coutinho de Macedo'

Rita de Cássia de Aquino²

${ }^{1}$ Centro Universitário Senac. São Paulo, SP, Brasil.

${ }^{2}$ Universidade São Judas Tadeu, Curso Mestrado em Ciências do Envelhecimento. São Paulo, SP, Brasil.

Correspondência / Correspondence

Rita de Cássia de Aquino

E-mail: rcaquino@uol.com.br

\section{Resumo}

$\mathrm{O}$ atendimento nutricional contribui para que o indivíduo seja agente de suas escolhas alimentares e consciente dos benefícios de uma alimentação saudável e deve ser pautado em documentos norteadores, tais como o Marco de Referência de Educação Alimentar e Nutricional para Políticas Públicas. Este artigo se propõe a refletir sobre o atendimento nutricional, discutindo conceitos relevantes como a educação alimentar e nutricional, os determinantes do padrão dietético, o aconselhamento e a forma como o Marco de Referência pode colaborar com esse processo. Os principais pontos de interface entre o Marco e o atendimento nutricional são a necessidade de compreender as interações e significados que determinam o comportamento alimentar, não devendo o atendimento enfatizar apenas os aspectos biológicos da nutrição. Destaca-se a postura adequada do profissional, como educador, que favorece a abertura do diálogo e contribui para o empoderamento, promoção do autocuidado e geração de autonomia no que tange ao comportamento alimentar. Considerando a complexidade das escolhas alimentares, instrumentos que colaborem com o aconselhamento e a educação alimentar e nutricional podem contribuir de forma significativa ao acesso democrático a uma alimentação saudável.

Palavras-chave: Educação Alimentar e Nutricional. Nutrição em Saúde Pública. Políticas Públicas. 


\section{Abstract}

Nutritional care contributes to the individual being an agent of his food choices, aware of the benefits of healthy eating. It should be guided by documents such as the Marco de Referência de Educação Alimentar e Nutricional para Políticas Públicas [Food and Nutrition Education Framework for Public Policies]. This article aims to reflect on nutritional care, discussing relevant concepts such as food and nutrition education, the determinants of dietary pattern, counseling, and how the Framework can contribute with this process. The main interface points between this document and nutritional care are the need to understand the interactions and meanings that determine feeding behavior, and care should not focus only on biological aspects of nutrition. Emphasis is placed on the professional's proper posture as an educator, which favors the opening of dialogue and contributes to empowerment, promotion of self-care and generation of autonomy concerning eating behavior. Considering the complexity of food choices, instruments that collaborate with counseling and food and nutrition education can significantly contribute to democratic access to healthy food.

Keywords: Food and Nutritional Education. Nutrition in Public Health. Public Policies.

\section{Introdução}

De acordo com a Política Nacional de Alimentação e Nutrição (PNAN), ${ }^{1}$ a Educação Alimentar e Nutricional (EAN) compõe o elenco de estratégias fundamentais para a promoção da alimentação adequada e saudável, sendo fundamental no processo de atendimento nutricional. E, apesar do progresso histórico nas ações no campo da EAN, constatou-se a necessidade de ampliar a discussão sobre as possibilidades, limites e modos como é realizada nos diversos ambientes e contextos. Assim, o Ministério do Desenvolvimento Social e Combate à Fome coordenou e publicou o Marco de Referência de Educação Alimentar e Nutricional para as Políticas Públicas, ${ }^{2}$ documento norteador que tem como objetivo promover um campo comum de reflexão e orientação da prática.

Na Epidemiologia das Doenças Crônicas, a alimentação é considerada importante fator etiológico modificável e uma das principais causas da obesidade. ${ }^{3}$ Estratégias que envolvam a alimentação como forma de promoção de saúde são imprescindíveis para a efetividade de políticas para a vida saudável ${ }^{4,5}$ e devem ser pautadas na prática da educação alimentar e nutricional (EAN), viabilizadas pelo atendimento nutricional. 
$\mathrm{O}$ atendimento nutricional pode ser definido como o ato de prestar assistência em relação à alimentação e nutrição de um indivíduo, grupo ou população. Tem como meta viabilizar condutas que venham promover um padrão alimentar que contribua com a qualidade de vida e que atenda às necessidades e recomendações nutricionais.

A demanda para o atendimento nutricional tem crescido nos últimos anos e ocupa importante papel na promoção de saúde, prevenção e tratamento de doenças. A busca por uma alimentação saudável tem levado a população a procurar por aconselhamento, a fim de rever seus hábitos alimentares e selecionar os alimentos consumidos.

É importante destacar que a educação alimentar e nutricional deve considerar as diversas dimensões da alimentação e elementos que compõem o comportamento alimentar. Muito se conhece sobre os aspectos que são relevantes e determinam o consumo, mas pouco se utiliza no processo de atendimento. Hábitos são influenciados por determinantes complexos e dinâmicos que interagem, e a alimentação deve ser entendida não apenas pela contribuição biológica, mas pelo conjunto das necessidades biopsicossociais que pode representar.

Assim, este artigo se propõe a refletir sobre o atendimento nutricional, apresentando e discutindo conceitos e pontos de intersecção sobre os temas de Educação Alimentar e Nutricional apresentados no Marco de referência, ${ }^{2}$ bem como os determinantes de consumo e os processos de aconselhamento nutricional nas quais se processam as ações do atendimento nutricional.

\section{Marco de Referência de Educação Alimentar e Nutricional}

Em 2012, o Ministério do Desenvolvimento Social e Combate à Fome (MDS) publicou o Marco de Referência de Educação Alimentar e Nutricional para Políticas Públicas, ${ }^{2}$ importante documento a ser utilizado em diversas esferas civis, públicas e acadêmicas no Brasil.

Considerando-se o campo de Segurança Alimentar e Nutricional (SAN), ${ }^{6}$ o documento busca um diálogo entre os diversos programas disponíveis, tendo como base práticas alimentares promotoras de saúde, que repensem a complexidade da articulação entre políticas públicas e o modo de fazer. ${ }^{7}$

A necessidade de publicar um documento como o Marco foi apontada por Santos, ${ }^{8}$ que enfatizou haver poucas referências que norteassem as políticas públicas: "a educação alimentar e nutricional está em todos os lugares e, ao mesmo tempo, em lugar nenhum” (p. 688). Porém, desde sua publicação, ainda se observa uma escassez de trabalhos acadêmicos e científicos que façam referência ao documento e que ressaltem sua relevância, especialmente no contexto do atendimento nutricional. 
O documento sugere a adoção da nomenclatura "Educação Alimentar e Nutricional", contraindicando os termos "Educação Alimentar" ou "Educação Nutricional" uma vez que estes, isolados, podem, no primeiro caso, reforçar apenas os aspectos relacionados ao alimento e alimentação e, no segundo, apenas os aspectos nutricionais. Essa indicação corrobora um amplo conceito de educação alimentar e nutricional que contempla desde o contexto no qual será realizada, os campos de conhecimento, abordagem educativa e o modelo pedagógico, até o espaço e o público a que se destina.

Observa-se que o Marco não fez referência ao termo "reeducação alimentar", muitas vezes utilizado no processo de aconselhamento nutricional e também no meio acadêmico. Santos ${ }^{9}$ destaca que não está muito clara, nas bases teóricas na literatura científica, a adequação conceitual deste termo. No entanto, ao traçar um paralelo entre o conceito de "educação alimentar e nutricional" estabelecido no Marco e a expressão "reeducação alimentar", infere-se que não seja adequada tal utilização. Isso porque o processo educativo presume prática contínua e permanente, implicando tomada de decisões e consequente transformação da realidade, considerando todas as interações e significados que compõem o comportamento alimentar. Esse é um percurso que não há como refazer, reconstruir ou reeducar, e sim fazer a partir de uma nova estrutura, construir a partir de um novo conhecimento ou educar a partir de novas vivências.

Sobre os campos de práticas da educação alimentar e nutricional (EAN), o Marco aponta que as mesmas podem ocorrer em diversos setores e devem observar os "princípios organizativos e doutrinários do campo no qual está inserida” (p. 24) e, enquanto Política Pública, a educação alimentar e nutricional pode ocorrer em diversas áreas da sociedade. Segundo Santos, ${ }^{9}$ as práticas desenvolvidas no campo da Nutrição Clínica e Ambulatorial são concebidas em um complexo contexto de atenção à saúde. É importante considerar que as atividades de atendimento nutricional, individualizadas ou em grupos, realizadas em ambulatórios das redes institucionais de serviço público e consultórios privados, devem ser pautadas nas recomendações do documento.

Considerando "alimentação uma prática social, resultante da integração das dimensões biológica, sociocultural, ambiental e econômica" (p. 31), o $\operatorname{Marco}^{2}$ destaca que a EAN requer uma abordagem integrada, e por esse motivo, profissionais de diversas áreas podem e devem desenvolver ações relacionadas. No entanto, quando envolvam indivíduos ou grupos com alguma doença, onde a EAN seja considerada um recurso terapêutico, as ações devem respeitar as especificidades regulamentadoras das categorias profissionais.

Em estudo de análise de produção científica brasileira sobre intervenções em educação alimentar e nutricional (EAN), Cervato-Mancuso et al. ${ }^{10}$ observaram que houve maior atuação do nutricionista nas intervenções que apresentaram como pretensão o tratamento de doenças, quando comparados aos estudos focados na promoção de saúde e alimentação saudável. Os autores inferem que a possível ampliação de atuação em EAN para outros profissionais, vislumbrada no Marco, pode-se dever ao 
fato de que nutricionistas não vinham desenvolvendo as práticas de forma suficientemente acessíveis às demandas da sociedade, sendo, portanto, indicada a abertura de ação para outros profissionais.

Sobre a formação dos profissionais da área da saúde, apontam-se desafios a serem superados, uma vez que são insuficientes os métodos de ensino relacionados à educação alimentar e nutricional (EAN) e observa-se pouca articulação entre o estudo da Ética, Filosofia, Sociologia e Antropologia e os aspectos biológicos da Alimentação e Nutrição. Além disso, há predominância da abordagem biomédica e de estruturas curriculares que não vislumbram que a abordagem de EAN aconteça de forma transversal entre os componentes curriculares. ${ }^{2}$

Alguns desses desafios foram apontados por $\mathrm{Boog}^{11}$ na formação do nutricionista, que destacou predomínio do estudo das ciências biológicas e desvalorização das ciências humanas. Segundo Navolar et al., ${ }^{12}$ a ciência da Nutrição, por estar inserida no modelo "biologicista" e com foco na doença e no risco, apresenta práticas interventivas restritivas que transformam a EAN em prescrições de alimentos e suplementos, desarticulando a abordagem do contexto social do sujeito com suas crenças e história.

Para Santos, ${ }^{13}$ a abordagem dos aspectos culturais da alimentação presente no Marco é extremamente relevante, pois permite um "alargamento das formas de ver e pensar a tríade do comer, alimentar e nutrir e como elas dialogam com as dimensões forjadas pela biomedicina, eixo central da Ciência da Nutrição desde seu nascimento" (p. 597).

Uma análise de publicações referentes a ações em Educação Alimentar e Nutricional (EAN) reconhece que há avanços na busca da integralidade do sujeito na sua dimensão biopsicosocial, com importante contribuição da Psicologia em contraponto ao modelo biomédico vigente. No entanto, ainda predomina a abordagem fragmentada entre os conhecimentos da área biomédica e das ciências humanas comportamentais, com abordagens pautadas em informações dietéticas, nutrientes e grupos alimentares. ${ }^{9,10}$

Frente a esses desafios, o $\operatorname{Marco}^{2}$ apresenta uma agenda pública voltada para a formação profissional, estudos e pesquisas na qual se destacam as ações para extensão, que valorizem a articulação entre saberes populares, comunidades tradicionais e conhecimento técnico-científico. Nesse contexto, a nova versão do Guia Alimentar para a População Brasileira ${ }^{14}$ foi elaborada, tendo em vista o atendimento aos princípios e diretrizes estabelecidos pelo Marco, configurando-se como instrumento para apoiar ações de educação alimentar e nutricional.

A publicação do Marco $^{2}$ é um importante passo para direcionar reflexões sobre EAN nos diversos setores da sociedade e campos de ação. No entanto, apresenta-se como um documento inacabado e em contínuo processo de construção, uma vez que haverá desdobramentos próprios que, a partir das novas experiências, aprendizados, princípios e saberes, poderão ser incorporados na prática do atendimento nutricional. 


\section{Determinantes do comportamento alimentar}

Segundo o $\operatorname{Marco,}^{2}$ a Educação Alimentar e Nutricional deve considerar "as interações e significados que compõem o comportamento alimentar" (p. 23) uma vez que o

[...] ato de comer, além de satisfazer as necessidades biológicas, é também fonte de prazer, socialização e expressão cultural, e as características do modo de vida contemporânea influenciam significativamente o comportamento alimentar (p.14).

No processo educativo do atendimento nutricional, que visa promover a autonomia para escolhas adequadas, é importante considerar que o comportamento alimentar é determinado por fatores pessoais, sociais, culturais, econômicos e ambientais e, principalmente, pela interação entre eles. A alimentação, comumente vinculada à promoção de saúde, muda segundo contextos históricos, a partir de diferentes formas de construção social, mudanças ambientais e tecnológicas na sociedade, que podem determinar o consumo alimentar. ${ }^{8,15}$

É no início da vida que os hábitos alimentares vão sendo formados, desde as primeiras experiências do aleitamento materno, até o processo de transição para uma alimentação geral, que será moldada pelas preferências inatas da criança, pelas decisões familiares e contexto social e afetivo em que esses são experimentados. ${ }^{16-19}$ É importante destacar a relevância da estrutura familiar, uma vez que o indivíduo está diariamente exposto a esses padrões e hábitos.

Segundo Rossi et al., ${ }^{19}$ os pais servem de modelo para o comportamento alimentar da criança, uma vez que têm papel fundamental no processo da aprendizagem e na exposição a padrões alimentares. Para Poulain, ${ }^{17}$ é na refeição familiar que "as crianças interiorizam as regras e valores da propriedade, do respeito aos outros, da partilha" (p. 177).

O conhecimento de que os indivíduos dispõem sobre a alimentação, seja ele científico ou popular, expresso por mitos, crenças e tabus, também determinam, em certa medida, o comportamento alimentar. Neste item, inserem-se as informações divulgadas pelos meios de comunicação, especialmente a televisão, que exercem influência na compra e consumo de alimentos. ${ }^{18-20}$

As características sensoriais dos alimentos como sabor, aparência, odor e textura agradáveis são os principais preditores da escolha alimentar, principalmente se associadas à memória de experiências anteriores. A depender do contexto social no qual os alimentos são apresentados e consumidos, pode haver uma associação de prazer, busca e repetição do alimento quando existe associação positiva de recompensa, ou seja, uma memória agradável associada ao consumo. Da mesma maneira, quando o consumo estiver associado a um evento de conflito, possivelmente haverá rejeição ao alimento. ${ }^{20}$ 
Dentre os determinantes do comportamento alimentar, também estão aqueles relacionados ao sistema de produção de alimentos, que correspondem ao conjunto de estruturas tecnológicas e sociais empregadas desde a coleta até a preparação culinária, ${ }^{21}$ o que Poulain ${ }^{17}$ define como "canais nos quais se deslocam os alimentos" (p. 49). Segundo o autor, "os alimentos não se movimentam sozinhos" (p. 49). O acesso físico e financeiro ao alimento, o desenvolvimento social, a produção de alimentos, os métodos de aquisição e as transformações culinárias definirão aquilo que o indivíduo irá consumir.

Fatores biológicos individuais, subdivididos em componentes fisiológicos, patológicos e genéticos, também determinam o comportamento alimentar. As necessidades nutricionais influenciarão na quantidade e qualidade da alimentação a ser consumida nos diferentes estágios da vida e condições fisiológicas ou patológicas. ${ }^{22}$

No Marco, $^{2}$ observa-se uma instigação para a necessidade de discutir e considerar os determinantes do comportamento alimentar no contexto de EAN. No entanto, a abordagem sobre este tema limita-se à descrição de que as escolhas alimentares são oriundas das dimensões individuais (aspectos subjetivos, conhecimento sobre a alimentação e percepções individuais) e coletivas (fatores econômicos, sociais e culturais). É relevante ampliar a discussão, uma vez que a educação alimentar e nutricional terá resultados mais eficazes, favorecendo a autonomia de escolha dos indivíduos, se articulada a estratégias que abranjam esses determinantes que envolvem o comportamento alimentar.

Para Cervato-Macuso et al., ${ }^{10}$ estudos de intervenção educativa que vierem a ser realizados após a publicação do Marco poderão apresentar resultados que contemplem estratégias mais inclusivas e problematizadoras, nas quais a pesquisa qualitativa seja fortalecida, evidenciando uma compreensão mais evidente da complexa rede dos fatores que determinam o comportamento alimentar.

\section{0 atendimento nutricional e 0 aconselhamento dietético}

$\mathrm{O}$ atendimento nutricional pode ser realizado em vários contextos e ambientes. Segundo a Resolução no 380/2005 do Conselho Federal de Nutricionistas (CFN), ${ }^{23}$ o atendimento é definido como "serviço de informação ou assistência prestado ao cliente ou paciente que necessite de orientações, informações ou cuidados alimentares e nutricionais específicos", e inclui a consulta nutricional. A consulta nutricional é definida como uma

[...] atividade realizada por nutricionista em unidade de ambulatório, ambiente hospitalar, consultório ou em domicílio para o levantamento de informações que possibilitem o diagnóstico nutricional e o conhecimento sanitário, a prescrição dietética e orientações individualizadas. ${ }^{23}$ 
O atendimento nutricional é um processo dinâmico e imprevisível, com diversas linguagens, que podem auxiliar no cuidado nutricional e ao mesmo tempo criar barreiras. O momento e o espaço onde é realizado deve favorecer o acesso aos saberes mútuos e partilha dos mesmos. ${ }^{7} \mathrm{O}$ profissional deve estar aberto para receber o indivíduo e criar um ambiente gentil e receptivo. É importante realizar inicialmente uma apresentação e colocar-se à disposição para o diálogo e valorizar a iniciativa de busca de atendimento. Assim, a empatia e a formação de vínculo são os passos iniciais para estabelecer uma relação de confiança entre os sujeitos envolvidos no processo.

No atendimento, outro ponto importante a ser considerado é a carga emocional dos indivíduos, pois trazem consigo expectativa, angústia e fragilidade que devem ser identificadas e respeitadas. Deve-se perceber sinais de nervosismo e ansiedade, observando atitudes como gestos, expressões faciais e corporais, além do timbre da voz e o excesso de informações ou de silêncio. É importante reduzir a intensidade desses sentimentos, que podem dificultar o estabelecimento do vínculo necessário. Após a redução da intensidade do primeiro contato, é importante ouvir a "queixa" individual, buscando compreender as razões da busca por atendimento e favorecendo o diálogo para gerar relatos verdadeiros que evite a omissão de informações. ${ }^{24}$

A relação com o indivíduo deve ser a prioridade no atendimento nutricional e em todas as ações profissionais. É preciso, antes de tudo, compreender o outro, conhecendo suas queixas e inquietações, permitindo o desencadeamento do atendimento. Por meio de estratégias e práticas adequadas, em conjunto com uma adequada formação humanística e no cuidado centrado na pessoa, o paciente poderá ser estimulado e encorajado a fazer escolhas mais saudáveis em relação à sua saúde.

Um dos protocolos previstos no atendimento nutricional é a realização da anamnese nutricional, que pode ser definida como a entrevista e o registro detalhado dos antecedentes fisiológicos, patológicos e socioeconômico-culturais do paciente e de seus familiares, com a finalidade de facilitar o diagnóstico nutricional. ${ }^{25}$ As informações obtidas a partir da anamnese devem servir como base para a problematização em busca de soluções para o tratamento, apontando-se as alternativas relacionadas aos hábitos alimentares e de vida de cada indivíduo.,24 No entanto, como reflexo do predomínio das ciências biológicas na formação dos profissionais de saúde, ${ }^{11,12}$ é natural observar nas anamneses uma atenção maior para os dados antropométricos, clínicos e dietéticos, estes mais focados nos dados quantitativos do consumo alimentar. O campo de investigação para elementos sociais, históricos e culturais que influenciam na alimentação é, em muitos casos, reduzido a apenas uma questão sobre a religião do indivíduo ou sobre sua etnia.

Um dos princípios para as ações de EAN estabelecidos no $\operatorname{Marco}^{2}$ trata da importância da abordagem do sistema alimentar na sua integralidade. Para isso, é importante ampliar a investigação no processo de anamnese de forma a identificar elementos que influenciam no comportamento alimentar ao longo de todo o processo alimentar. 
Na prática, pouco se questiona e se identifica sobre os fatores que influenciam nas escolhas alimentares representadas pelas interações e significados que compõem o comportamento e o sistema alimentar na sua integralidade. Esse evento foi observado no estudo de Cervato-Mancuso et al. ${ }^{10}$ Os autores relataram que, dentre os estudos de intervenção educativa analisados, a maioria remetia aos indicadores quantitativos, especialmente sobre consumo alimentar e verificação antropométrica, ficando em segundo plano as questões comportamentais.

Além da anamnese, faz parte do processo de atendimento nutricional o aconselhamento dietético, definido por Rodrigues et al. ${ }^{26}$ como

[...] uma abordagem de educação nutricional, efetuada por meio do diálogo entre o cliente portador de uma história de vida - que procura ajuda para solucionar problemas de alimentação - e o nutricionista, preparado para analisar o problema alimentar no contexto biopsicossociocultural da pessoa, que a auxiliará a explicitar os conflitos que permeiam o problema, a fim de buscar soluções que permitam integrar as experiências de criação de estratégias para o enfrentamento dos problemas alimentares na vida cotidiana, buscando um estado de harmonia compatível com a saúde (p. 127).

No entanto, na prática, o aconselhamento é frequentemente realizado de forma prescritiva, limitada ao que deve ser feito, sem considerar as dimensões que afetam o comportamento. Trata-se de uma abordagem tradicional da educação fundamentada na transmissão oral e de conhecimento entre alguém que detém o conhecimento e o transfere àquele que não o tem. O indivíduo não é o foco de atendimento, mas sim a doença que ele apresenta, que o fragmenta e reduz a responsabilidade da ação. ${ }^{26}$ Para Boog, ${ }^{11}$ esse modelo é o que se conceitua como "orientação nutricional”. Segundo a autora, a "orientação" difere do termo "educação" pois, embora inserida no processo de educação, a orientação faz parte de um escopo de ações mais restritas, limitadas ao objetivo de instruir em casos de uma emergente necessidade de mudança, quando, por exemplo, o indivíduo recebe o diagnóstico de uma doença para a qual há necessidade de alterações alimentares imediatas.

A arte do aconselhamento não deve se limitar a "proibir" e "permitir". O aconselhamento deve ser pautado em um processo dinâmico de educação, respeitando-se os valores biopsicossociais do indivíduo. Considerando os conceitos adotados por Rodrigues et al., ${ }^{26}$ o aconselhamento visa promover apoio técnico e emocional ao indivíduo no desenvolvimento de sua capacidade pessoal em realizar suas próprias escolhas alimentares, permitindo uma relação educativa diferenciada e voltada para a tomada de decisões e atitudes situadas no contexto de suas vivências.

Segundo o Marco, ${ }^{2}$ a forma como a comunicação é desenvolvida é fundamental para a obtenção de resultados que favoreçam as escolhas alimentares adequadas e que ultrapassem os limites da transmissão verbal de informações. A comunicação no contexto da educação alimentar e nutricional (EAN) deve ser pautada na escuta ativa e próxima, no reconhecimento e valorização das diferentes formas de saberes e práticas e na busca de soluções contextualizadas. Assim, 
destaca a importância da valorização do respeito às histórias individuais e o reconhecimento da legitimidade dos saberes de diferentes naturezas, sem expressão de julgamento imposta pelo profissional. Segundo Paulo Freire, ${ }^{27}$ o processo educativo que favorece a autonomia exige do educador o respeito aos saberes dos educandos.

A atitude de escuta pressupõe a capacidade do profissional em propiciar um espaço para que o indivíduo possa expressar aquilo que sabe, pensa e sente em relação a suas escolhas alimentares e responder a suas reais expectativas, dúvidas e necessidades. É importante motivá-lo a expor suas necessidades e dificuldades para a adoção de práticas e hábitos de vida saudáveis, pois é nesta relação entre o educador e educando que se estabelece a possibilidade de uma experiência de problematização do comportamento alimentar e a oportunidade de transformá-la em solução. ${ }^{28,29}$

No processo de aconselhamento dietético, é necessário compreender os diversos significados que o indivíduo atribui a suas limitações e possibilidades em resolver os problemas relacionados à sua alimentação. Para tanto, é fundamental desenvolver uma comunicação competente, isto é, informações apropriadas às necessidades do indivíduo, além da adequação da linguagem empregada para favorecer a compreensão do conteúdo. ${ }^{30}$

O profissional que se dedica ao aconselhamento dietético necessita desenvolver características pessoais e profissionais que facilitem o processo educativo e o "empoderamento" para escolhas alimentares adequadas. Um dos princípios do Guia Alimentar para a População Brasileira, ${ }^{14}$ respaldado pelo Marco, é o reconhecimento de que os guias devem ampliar a autonomia nas escolhas alimentares, que as pessoas possam buscar as mudanças em si mesmas e terem condições de governar e conduzir suas próprias vidas, fazendo escolhas que promovam a melhora da qualidade de vida.

Empatia, tolerância, compreensão e flexibilidade são, portanto, imprescindíveis. Na abordagem, o indivíduo deve se sentir acolhido e compreendido, e não julgado por suas atitudes alimentares. ${ }^{24}$ Nesse contexto, o $\operatorname{Marco}^{2}$ aponta para a necessidade de ampliar a discussão sobre o processo de comunicação em EAN, desenvolvendo novos instrumentos teóricos e metodológicos que abordem questões relevantes como compartilhamento de saberes e construção de soluções.

O $\operatorname{Marco}^{2}$ infere que a forma de comunicação influencia diretamente nos resultados do processo educativo e por isso sugere que a mesma seja pautada em uma escuta ativa e próxima, no reconhecimento de diferentes saberes e práticas, na formação de vínculo entre os diferentes sujeitos e na busca de soluçóes contextualizadas.

As práticas educativas devem procurar seguir os princípios para as ações de educação preconizadas pelo Marco, ${ }^{2}$ que sugere a promoção do autocuidado e a autonomia como principais caminhos para garantir o envolvimento do indivíduo nas ações de EAN, gerando situações de reflexão sobre atitudes e consequente busca de soluções pautadas na problematização. 
A abordagem teórico-pedagógica que vislumbra a ênfase na dialogicidade e autonomia do sujeito, com utilização de recursos problematizadores, é fundamentada no pensamento de Paulo Freire. ${ }^{27}$ Para o educador, "nas condições verdadeiras de aprendizagem os educandos vão se transformando em reais sujeitos da construção e da reconstrução do saber ensinado, ao lado do educador, igualmente sujeito do processo" (p. 26). Isso somente é possível pelo diálogo horizontal entre os agentes do processo educativo, no qual existem respeito e mútua busca por alternativas para a resolução de problemas.

Assim, é necessário desenvolver no indivíduo valores positivos em relação a seus hábitos, que contribuam com sua autodescoberta e reconhecimento do que pode estar contribuindo com suas atitudes em relação a suas escolhas alimentares. Toral ${ }^{30}$ coloca, em seu estudo sobre comportamento alimentar em adolescentes, que um importante fator a ser desenvolvido durante o aconselhamento é a autoeficácia do indivíduo, isto é, o desenvolvimento da confiança que ele tem em si mesmo em relação à sua habilidade para fazer escolhas alimentares saudáveis.

Um dos desafios apresentados no processo de aconselhamento dietético é a adoção da comida e do alimento como referências, uma vez que

[...] as pessoas, [...], não se alimentam de nutrientes, mas de alimentos e preparações escolhidas e combinadas de uma maneira particular, com cheiro, cor, temperatura, textura e sabor, se alimentam também de seus significados e dos aspectos simbólicos. ${ }^{2}$ (p. 26).

É fundamental, assim, atender ao princípio da valorização da culinária enquanto prática emancipatória, estabelecido no $M a r c o,{ }^{2}$ pois facilita a reflexão e o exercício das dimensões sensoriais, cognitivas e simbólicas da alimentação. O desenvolvimento de habilidades culinárias é estimulado no Guia Alimentar para a População Brasileira ${ }^{14}$ como uma prática fundamental para geração de autonomia e ampliação das possibilidades de escolhas para uma alimentação à base de alimentos in natura.

Estudo realizado por Castro et al. ${ }^{31}$ identificou que, por meio de culinária, foi possível proporcionar aos participantes uma experiência de vivência e reflexão sobre as relações entre alimentação, cultura e saúde, resultando na instrumentalização para as escolhas e práticas adequadas à promoção da alimentação saudável. Os autores apontam ser factível a utilização da culinária como eixo estruturante de estratégia educativa, pois desenvolve a autonomia para as escolhas, além de envolver o sujeito como protagonista do processo.

Como resultado desta reflexão, vislumbra-se um formato de espaço e estrutura física para o atendimento nutricional nos quais, além de salas equipadas com instrumentos para antropometria e computadores, deveriam ser instaladas cozinhas onde poderiam ser desenvolvidas preparações saborosas e ao mesmo tempo saudáveis. ${ }^{31}$ 
Embora o $\operatorname{Marco}^{2}$ faça referência ao "uso de recursos educacionais" e que seja sabida a importância da escolha de materiais adequados e estratégias educativas adaptadas segundo recursos físicos e humanos, o documento não infere sobre materiais ou recursos didáticos que possam ser utilizados nos processos educativos.

Para Santos, ${ }^{13}$ o Marco traz à tona a necessidade de reflexão sobre como pensar na formação de profissionais que possam atuar em práticas educativas que acontecem em um mundo real no qual os eventos não podem ser controlados, tais como os experimentos laboratoriais. O desafio é construir indicadores quantitativos e qualitativos pertinentes ao campo que envolve a educação, exigindo dos pesquisadores a "disposição de lidar com o ineditismo da experiência humana e estar implicados com sua prática de pesquisa” (p. 599).

No processo de aconselhamento, é preciso assumir o desafio de exercer o papel de educador, contemplando a preocupação com as representações sobre o comer e a comida, a busca de autonomia e a aplicação de abordagens problematizadoras, além da abertura de diálogo e construção coletiva de uma proposta dietética na qual o indivíduo seja o agente de sua transformação e permita a adoção de comportamentos que contribuam para a sua saúde. ${ }^{9,11,13}$

\section{Consideraçóes finais}

A Educação Alimentar e Nutricional se apresenta como uma das estratégias fundamentais para a prevenção e controle dos problemas alimentares e nutricionais contemporâneos, sendo fundamental no processo de atendimento nutricional. No entanto, o atendimento e o aconselhamento dietético são práticas complexas e desafiadoras que exigem extrapolar a dimensão da informação correta e ampliar sua ação para o contexto biológico, psicológico, social e cultural do indivíduo. A riqueza dessa prática está em promover mudanças no pensar, no sentir e no agir.

Nesse contexto, o Marco de Referência de Educação Alimentar e Nutricional para Políticas Públicas ${ }^{2}$ vem propiciar maior reflexão sobre a importância do atendimento nutricional na promoção da saúde da população brasileira e, segundo o documento, "a promoção da alimentação adequada e saudável é a expressão da cidadania e fator protetor da vida" (p. 36).

É preciso reduzir o hiato que existe entre as informações disponíveis sobre alimentação e o estado de saúde e nutrição da população. Se, por um lado, as informações sobre alimentação saudável têm sido amplamente divulgadas, por outro, a epidemia da obesidade e de outras doenças crônicas vem apresentando progressivo aumento em todas as idades. Tal descompasso induz ao questionamento de como estratégias de prevenção vêm sendo aplicadas e quais favorecem efetivamente a incorporação de novos hábitos alimentares. Dentre as estratégias reconhecidas, o aconselhamento dietético vem sendo apontado como uma prática eficaz e fundamental para a adoção de atitudes voltadas para o cuidado que o indivíduo tem com si próprio. 
Porém, para uma análise mais efetiva da aplicação dos princípios do Marco no atendimento nutricional, é necessário ampliar a publicação de estudos que adotem descrição metodológica que apresentem os elementos suficientes para a compreensão e interpretação de todo o percurso educativo, incluindo agentes, estratégias educativas, sistemas de avaliação e observação dos aspectos comportamentais.

Uma orientação bem planejada e individualizada deve permitir que o indivíduo seja agente de suas escolhas, consciente dos benefícios de hábitos saudáveis e, gradativamente, tenha uma alimentação que permita promover a saúde e reduzir o risco de doenças.

$\mathrm{O}$ atendimento nutricional implica compromisso e deve ser fornecido de forma presencial, continuada, sistematizada e documentada, com a participação ativa do indivíduo, para obtermelhor qualidade de vida. Espera-se que o Marco venha a ser um instrumento que contribua com o objetivo de garantir o acesso à alimentação saudável.

\section{Colaboradores}

Macedo IC e Aquino RC realizaram em conjunto a redação do texto e a revisão crítica final.

Conflito de interesses: as autoras declaram não haver conflitos de interesses.

\section{Referências}

1. Brasil. Ministério da Saúde. Política Nacional de Alimentação e Nutrição. Brasília: Ministério da Saúde; 2012.

2. Brasil. Ministério do Desenvolvimento Social e Combate à Fome. Marco de referência de educação alimentar e nutricional para políticas públicas. Brasília: Ministério da Saúde; 2012.

3. World Health Organization. Diet, nutrition and the prevention of chronic diseases. Geneve: WHO; 2003. WHO technical report series, 916.

4. Cotta RMM, Reis RS, Batista KCS, Dias G, Alfenas RC, Castro FAF. Hábitos e práticas alimentares de hipertensos e diabéticos: repensando o cuidado a partir da atenção primária. Rev Nutr. 2009; 22(6):823-835.

5. Vieira CM, Cordeiro SN, Magdaleno Júnior R, Turato ER. Significados da dieta e mudanças de hábitos para portadores de doenças metabólicas crônicas: uma revisão. Cienc Saúde Colet. 2011; 16(7):3161-3168.

6. Brasil. Conselho Nacional de Segurança Alimentar e Nutricional. Princípios e diretrizes de uma Política de Segurança Alimentar. Brasília: CONSEA; 2004.

7. Schneider OMF, Neves AS. Conversas sobre formar fazer a nutrição: as vivências e percursos da Liga de Segurança Alimentar e Nutricional. Interface. 2014; 18(4S):187-194. 
8. Santos LA. Educação nutricional no contexto de promoção de práticas alimentares saudáveis. Rev Nutr. 2005; 18(5):681-692.

9. Santos LA. O fazer educação alimentar e nutricional: algumas contribuições para reflexão. Cienc Saúde Colet. 2012; 17(2):453-462.

10. Cervato-Mancuso AM, Vincha KRR, Santiago DA. Educação Alimentar e Nutricional como prática de intervenção: reflexão e possibilidades de fortalecimento. Physis 2016; 26(1):225-249.

11. Boog MCF. Educação nutricional: passado, presente e futuro. Rev Nutr.1997; 10(1):5-19.

12. Navolar TS, Tesser CDS, Azevedo E. Contribuições para a construção da nutrição complementar integrada. Interface - Comunic Saúde Educ. 2012; 16(41):515-527.

13. Santos LA. Avanços e desdobramentos do marco de referência da educação alimentar e nutricional para as políticas públicas no âmbito da universidade e para os aspectos culturais da alimentação. Rev Nutr. 2013; 26(5):595-600.

14. Brasil. Ministério da Saúde. Guia alimentar para a população brasileira. Brasília: Ministério da Saúde; 2014.

15. Garcia RWD. Representation on food intake and its implications in nutritional investigations: qualitative study with subjects submitted to dietary prescriptions. Rev Nutr. 2004; 17(1):15-28.

16. Poulain JP, Proença RPC. O espaço social alimentar: um instrumento para o estudo dos modelos alimentares. Rev Nutr. 2003; 19(3):245-256.

17. Poulain JP. Sociologias da alimentação: os comedores e o espaço social alimentar. Florianópolis: Editora UFSC; 2004.

18. Quaioti TC, Almeida SS. Determinantes psicobiológicos do comportamento alimentar: uma ênfase em fatores ambientais que contribuem para a obesidade. Psicol USP 2006; 17(4):193-211.

19. Rossi A, Moreira EAM, Rauen MS. Determinantes do comportamento alimentar: uma revisão com enfoque na família. Rev Nutr. 2008; 21(6):739-748.

20. Viana V, Santos PL, Guimarães MJ. Comportamento e hábitos alimentares em crianças e jovens: uma revisão da literatura. Psicol Saúde e Doenças 2008; 9(2):209-231.

21. Fonseca BF, Souza TSN, Frozi DS, Pereira RA. Modernidade alimentar e consumo de alimentos: contribuições sócio-antropológicas para a pesquisa em nutrição. Cienc Saude Colet. 2011; 16(9):3853-3862.

22. Jamori MM, Proença RPC, Calvo MCM. Determinantes de escolha alimentar. Rev Nutr. 2008; 21(1):63-73.

23. Brasil. Conselho Federal de Nutricionistas. Resolução CFN 380, de 28 de dezembro de 2005. Dispõe sobre a definição das áreas de atuação do nutricionista e suas atribuições, estabelece parâmetros numéricos de referência, por área de atuação, e dá outras providências. Diário Oficial da União 10 jan. 2006; 1(7):66-71. Retificada Diário Oficial da União 10 ago. 2006; 1(153):52.

24. Aquino RC, Philippi ST. Nutrição clínica: estudos de casos comentados. Barueri: Manole; 2009. 
25. Guimarães AF, Galante AP. Anamnese nutricional e inquéritos dietéticos. In: Rossi L, Caruso L, Galante AP. Avaliação nutricional: novas perspectivas. Rio de Janeiro: Guanabara Koogan; 2015.

26. Rodrigues EM, Soares FPTP, Boog MCF. Resgate do conceito de aconselhamento no contexto do atendimento nutricional. Rev Nutr. 2005; 18(1):119-128.

27. Freire P. Pedagogia da autonomia. São Paulo: Paz e Terra; 1996.

28. Figueiras SL, Deslandes SF. Avaliação das ações de aconselhamento: análise de uma perspectiva de prevenção centrada na pessoa. Cad Saúde Pública 1999; 15(Sup. 2):121-131.

29. Sales CMB, Figlie NB. Revisão de literatura sobre a aplicação da entrevista motivacional breve em usuários nocivos e dependentes de álcool. Psicol Estud. 2009; 14(2):333-340.

30. Toral N. Estágios de mudança de comportamento e sua relação com o consumo alimentar de adolescentes [dissertação]. São Paulo: Universidade de São Paulo, Faculdade de Saúde Pública; 2006.

31. Castro IRR, Souza TSN, Maldonado LA, Caniné ES, Rotenberg S, Gugelmin SA. A culinária na promoção da alimentação saudável: delineamento e experimentação de método educativo dirigido a adolescentes e profissionais das redes de saúde e de educação. Rev Nutr. 2007; 20(6):571-588.

Recebido: 07 de maio, 2017

Revisado: 26 de setembro, 2017

Aceito: 11 de janeiro, 2018 
